\title{
BMJ Open Music intervention for sleep quality in critically ill and surgical patients: a meta-analysis
}

\author{
Ellaha Kakar (D) , ${ }^{1}$ Esmée Venema, ${ }^{2}$ Johannes Jeekel, ${ }^{3}$ Markus Klimek (D) , ${ }^{4}$ \\ Mathieu van der Jagt $^{5}$
}

To cite: Kakar E, Venema E, Jeekel J, et al. Music intervention for sleep quality in critically ill and surgical patients: a meta-analysis. BMJ Open 2021;11:e042510. doi:10.1136/ bmjopen-2020-042510

- Prepublication history and supplemental material for this paper is available online. To view these files, please visit the journal online (http://dx.doi org/10.1136/bmjopen-2020042510).

EK and EV are joint first authors.

Received 07 July 2020

Revised 19 January 2021

Accepted 13 April 2021

Check for updates

(c) Author(s) (or their employer(s)) 2021. Re-use permitted under CC BY. Published by BMJ.

${ }^{1}$ Department of Surgery and Intensive Care Unit, Erasmus MC, Rotterdam, The Netherlands ${ }^{2}$ Maastricht University, Maastricht, The Netherlands ${ }^{3}$ Department of Surgery and Neuroscience, Erasmus MC, Rotterdam, The Netherlands ${ }^{4}$ Department of Anaesthesiology, Erasmus MC, Rotterdam, The Netherlands

${ }^{5}$ Department of Intensive Care Unit, Erasmus MC, Rotterdam, The Netherlands

Correspondence to

Ellaha Kakar;

e.kakar@erasmusmc.nl

\section{ABSTRACT}

Objective Sleep disruption occurs frequently in hospitalised patients. Given the potential of music intervention as a non-pharmacological measure to improve sleep quality, we aimed to assess and quantify current literature on the effect of recorded music interventions on sleep quality and quantity in the adult critical care and surgical populations.

Design Systematic review and meta-analysis.

Data sources Embase, MEDLINE Ovid, Cochrane Central, Web of Science and Google Scholar.

Eligibility criteria for studies Randomised controlled trials assessing the effect of music on sleep quality in critically ill and surgical patients.

Methods The electronic databases were systematically searched from 1 January 1981 to 27 January 2020. Data were screened, extracted and appraised by two independent reviewers. Primary outcomes were sleep quality and quantity, assessed with validated tools. The Preferred Reporting Items for Systematic Reviews and Meta-Analyses guidelines were followed. Random effects meta-analysis was performed, and pooled standardised mean differences (SMDs) with $95 \% \mathrm{Cls}$ were reported. Results Five studies (259 patients) were included in qualitative (risk of bias) and quantitative analysis (meta-analysis). Pooled data showed a significant effect of recorded music on subjective sleep quality in the critical care and surgical population (SMD $=1.21(95 \%$ Cl 0.50 to 1.91), $p<0.01$, excluding one non-English study; SMD $=0.87$ ( $95 \% \mathrm{Cl} 0.45$ to 1.29 ), $\mathrm{p}<0.01$ ). The SMD of 1.21 corresponded to a $27.1 \%(95 \% \mathrm{Cl} 11.2$ to $42.8)$ increase in subjective sleep quality using validated questionnaires. A significant increase in subjective sleep quantity of $36 \mathrm{~min}$ was found in one study. Objective measurements of sleep assessed in one study using polysomnography showed significant increase in deeper sleep stage in the music group.

Conclusions Recorded music showed a significant improvement in subjective sleep quality in some critical care and surgical populations. Therefore, its use may be relevant to improve sleep, but given the moderate potential for bias, further research is needed.

PROSPERO registration number CRD42020167783.

\section{INTRODUCTION}

Sleep disruption is common in hospitalised patients. ${ }^{1-4}$ A large cross-sectional study in

\section{Strengths and limitations of this study}

- This is the first comprehensive systematic review that studies the effect of music on sleep quality in critically ill and surgical patients.

- Patient-reported outcomes, which are of increasing interest and of clinical relevance, are assessed in qualitative and quantitative analyses.

> Only data from randomised controlled trials are included.

- The risk of bias is moderate to high and the heterogeneity is high, which makes it difficult to draw definite conclusions.

the Netherlands found that sleep quality and quantity were significantly affected in hospitalised patients compared with patients' habitual sleep at home. ${ }^{5}$ Sleep disruption is prevalent in almost all surgical and critically ill patients. ${ }^{5-8}$ Important preventable factors associated with sleep disruption are pain, noise, anxiety, stress, sedative and analgesic medication usage, immobility and severity of illness. ${ }^{159-12}$

In critically ill and surgical patients, poor sleep has been associated with postoperative delirium, cardiovascular events in highrisk patients, postoperative fatigue (leading to prolonged immobility), altered mental status, ${ }^{13-16}$ prolonged mechanical ventilation, cognitive impairment, altered immune function and long-term psychological comorbidities. ${ }^{17}$ Thus, improving sleep promotes good health by improving emotional (anxiety and stress) ${ }^{18}$ and possibly physical recovery. This is especially important for intensive care unit (ICU) and surgical patients given the loss of tissue integrity. ${ }^{19}{ }^{20}$ Interventions in the hospital to improve sleep quality mainly include pharmacological interventions, such as benzodiazepines. ${ }^{21-25}$ The Clinical Practice Guidelines for the Prevention and Management of Pain, Agitation/Sedation, Delirium, Immobility, and Sleep Disruption 
in adult patients in the ICU strongly recommend avoiding these drugs, given their potential to induce delirium and substance dependence. ${ }^{17} 2627$ Therefore, nonpharmacological measures are advised to prevent sleep disruption in hospitalised patients. ${ }^{17}$ 28-36

Recently, several studies have shown a significant positive effect of perioperative music interventions on anxiety, pain, sedative and analgesic medication requirement, and neurohormonal stress response ${ }^{37-39}$ Also, in the ICU population associations have been made between music interventions and decreased serum cortisol levels, positive effects on state anxiety, reduction in respiratory rate and systolic blood pressure, and decrease in sedative and analgesic requirement in mechanically ventilated patients. ${ }^{40} 41$

Given the potential of music intervention as a nonpharmacological measure to improve sleep quality, we aimed to assess and quantify current literature on the effect of perioperative recorded music interventions on sleep quality and quantity in the adult critical care and surgical populations.

\section{MATERIALS AND METHODS Protocol and registration}

This systematic review and meta-analysis follows the Preferred Reporting Items for Systematic Reviews and Meta-Analyses guidelines (online supplemental file 1) and was registered in the PROSPERO database (https:// www.crd.york.ac.uk/PROSPERO, as record number CRD42020167783). ${ }^{42}$

\section{Search strategy}

A systematic search was performed together with a dedicated biomedical information specialist in the Embase, MEDLINE Ovid, Cochrane Central, Web of Science and Google Scholar databases using a standardised protocol, ${ }^{43}$ including articles between 1 January 1981 (the year in which the first minimally invasive surgical intervention was performed, in order to avoid old literature which is not compatible with the current standard perioperative pain practice) and 27 January 2020. The search strategy included terminologies related to: sleep quality/ insomnia, including sleep architecture (eg, rapid eye movement (REM) sleep) and music (eg, music therapy). The full search strategy per database is given in online supplemental file 2.

\section{Study screening and selection}

Two reviewers $(\mathrm{EK} / \mathrm{EV})$ independently screened results of the search strategy on title/abstract to confirm adherence to the eligibility criteria. Studies were eligible when they investigated (P) critically ill and/or surgical patients aged 18 years or older receiving a (I) recorded music intervention $(\mathrm{C})$ compared with a control group in order to assess the effect on $(\mathrm{O})$ sleep quality and quantity in (S) a randomised controlled trial (RCT). PICOS is a mnemonic used in evidence-based medicine and stands for, respectively, patient, intervention, control, outcomes and study type. ${ }^{44}$ Critically ill populations included patients admitted to ICU or cardiac care unit (CCU), since CCU patients were also considered to have (potential) vital organ failure. Other eligibility criteria were: full text available and the study included in-hospital patients. Since, the above-mentioned studies included patients who have the following in common; in-hospital patients and a compromised physiology (eg, by performing an intervention, surgical procedure or having critical illness), which both have impact on the patients comfort and thus sleep, the data were suitable to be pooled. Studies involving live music performance were excluded, since live music performance consists of two interventions: the music and the interaction with the musician. Music intervention was defined as the use of recorded music consisting of melody, harmony and rhythm. Nature sounds were only included when used in addition to music. If studies compared music with multiple groups, the group without music most similar to the music group was chosen as control group (eg, if groups were 'scheduled rest' and 'standard care', 'scheduled rest' was chosen as control group if the music group also received a resting period). The data of the extra arms were therefore not used in this study. Fulltext articles were discussed for admissibility. All disagreements between reviewers were resolved by discussion.

\section{Data collection process and items}

Data were extracted and checked by the same two reviewers $(\mathrm{EK} / \mathrm{EV})$ independently according to a predesigned dataset. The following study characteristics were extracted: author, year of publication, study type, country of study, reason for admission (eg, surgery type), sample size, age (mean and SD), gender (\% male), severity of disease of included patients, type and timing of sleep assessment, timing of the intervention, setting (eg, surgical ward, ICU and CCU), method of intervention delivery (eg, headphones and CD player), frequency and duration of the intervention, total duration of the intervention, music choice (eg, patient or researcher selected), type of control group and number of participants in the intervention and control group. Primary outcome measures were sleep quality and quantity measured after intervention or at the end of study period for both the control and intervention groups. If a study applied music intervention and collected data at multiple time points, only the final time point was used, since music could have a cumulative effect.

\section{Risk of bias assessment}

Risk of bias within studies was assessed independently by two reviewers (EK/EV) using the Cochrane Risk of Bias tool for RCTs. ${ }^{45}$ Incomplete data of $\geq 10 \%$ due to drop-out (attrition bias) was considered as high risk. If the study protocol was not available, the risk for selective reporting was considered as unclear. Publication bias was assessed by creating funnel plots, and the risk of bias across studies of the effect of music on sleep quality was assessed using 
the Grading of Recommendations, Assessment, Development and Evaluations (GRADE) criteria. ${ }^{45} 46$

\section{Statistical analysis}

Descriptive statistics were presented as means and their $\mathrm{SD}$, counts $(\mathrm{N})$ and percentages (\%). For categorical age groups (eg, age $20-50$ years=eight patients, 51-60 years=seven patients), an approximation of the mean was calculated by taking the central point of the range as the mean for each category and pooling these means for each trial separately, weighted to the sample size. The overall $\%$ of males in this review was calculated by averaging the $\%$ of males weighted to the sample size. If ranges were provided, these were used to calculate approximations for the SD by calculation one-fourth of the range of the data ((maximum-minimum) $/ 4) .{ }^{47}$ Studies were included for quantitative analysis if mean values and SDs of the sleep scores were reported. The primary analysis included meta-analysis for sleep quality and quantity. Standardised mean differences (SMDs) were calculated as summary statistics of the main outcome, and a random effects model was used to calculate the overall effect of music on sleep quality and quantity, accounting for between-study heterogeneity. The between-study variance was estimated using the restricted maximum likelihood method. The level of heterogeneity was assessed using the $\mathrm{I}^{2}$ statistic. For clinical interpretation, the effect of the music intervention on sleep quality scores was expressed as a relative percentage increase, using a back-transformation of the acquired SMD that was described by the Cochrane Handbook for Systematic Reviews. ${ }^{45}$ For this calculation, the SDs of the control groups were pooled to approximate the amount of variation in our patient population. Additionally, the SD of a study with a large sample assessing sleep was used to make a more accurate approximation of the amount of variation. Data were analysed using $\mathrm{R}$ V.3.6.3, and a two-sided $p$ value of $<0.05$ was considered statistically significant. To calculate the increase in subjectively measured sleep quality based on the pooled SMD of the meta-analysis, a back-transformation was applied to the sleep quality as described by the Cochrane Handbook for Systematic Reviews. ${ }^{45}$ For this back-transformation, the SDs of sleep were estimated by pooling the SDs of the control groups for the different assessment tools separately.

\section{Patient and public involvement}

No patients involved.

\section{RESULTS}

The search yielded 2127 articles leaving 1179 articles after removal of duplicates and 1146 articles after removal of articles before the year 1981. Title and abstract screening resulted in 10 articles for full-text assessment. Five studies were excluded for the following reasons: full text not available, use of guided imaging in combination with music, use of nature sounds and use of multiple interventions.
Subsequently, a total of five RCTs ${ }^{48-52}$ were included in the qualitative and quantitative analysis (259 patients) (figure 1).

\section{Study characteristics}

A detailed overview of patient and music intervention characteristics is presented in tables 1 and 2. Two studies were conducted at the ICU, two studies were conducted at the CCU and one study was performed in the surgical ward directly after ICU discharge. Apart from this study (Zimmerman et $a \tilde{l}^{2}$ ), no other surgical papers were found. The mean age of the study population was 62.4 years with a predominance of males $(65.8 \%)$. Hansen $e t$ $a l^{t 8}$ and $\mathrm{Su} e t a \tilde{l}^{11}$ conducted the study at the ICU. The study of Su et $a \tilde{l}^{1}$ included medical ICU patients, with an Acute Physiology and Chronic Health Evaluation II score of $\leq 25$, and the study of Hansen $e t a l^{48}$ included surgical $(59 \%)$ and medical $(41 \%)$ ICU patients. Cheraghi $e t a l^{49}$ and Ryu et $a l^{\tilde{0} 0}$ conducted their study at the CCU, respectively, including acute coronary syndrome (ACS) and percutaneous transluminal coronary angiography (PTCA) patients. The study of Zimmerman et $a \bar{l}^{2}$ was performed in the nursing ward after coronary artery bypass graft $(\mathrm{CABG})$ surgery. Since the abstract of the paper of Cheraghi et $a t^{t 9}$ was available in English, the study was included after title/abstract screening. The full text version was only available in the Persian language; the paper was translated by a statistician in the Erasmus MC with Persian as mother language. The study was included in this review due to acceptable methodology, which was not different than the other included studies.

Sleep quality was measured using the RichardsCampbell Sleep Questionnaire (RCSQ; 40\%), the Verran and Snyder-Halpern Sleep Scale (VSH; 40\%) and the Pittsburgh Sleep Quality Index (PSQI; 20\%). Online supplemental file 3 gives an overview of the previously mentioned sleep questionnaire characteristics. Only in the study of Ryu et al sleep quantity was measured using patient questionnaires. In the study of Su et al, ${ }^{51}$ sleep quality and quantity was also measured objectively, using the polysomnography (PSG). PSG is currently the golden standard in objectively measuring sleep variables, including total sleep time (TST), wake after sleep onset, sleep onset latency, REM latency, sleep efficiency, arousal index and percentage of TST spent in each sleep stage (N1, N2, N3 and REM). ${ }^{53}$

Music was mostly described as being soothing, sleep inducing or sedating $(60 \%) .{ }^{48} 50$ Music was selected by the researcher in four of the five studies $(80 \%)$; only Zimmerman et a $\tilde{l}^{2}$ provided the option of choice between five audiotapes according to the patients' preference (table 2). Music was mostly administered using headphones or earphones $(60 \%)$. Duration of the intervention was on average $40 \mathrm{~min}$ per session and ranged from 30 to $53 \mathrm{~min}$ per day. Cheraghi et $a t^{49}$ administered the music intervention during three consecutive evenings, just before bedtime, after admission to the CCU, and assessed sleep at baseline and every morning after an 


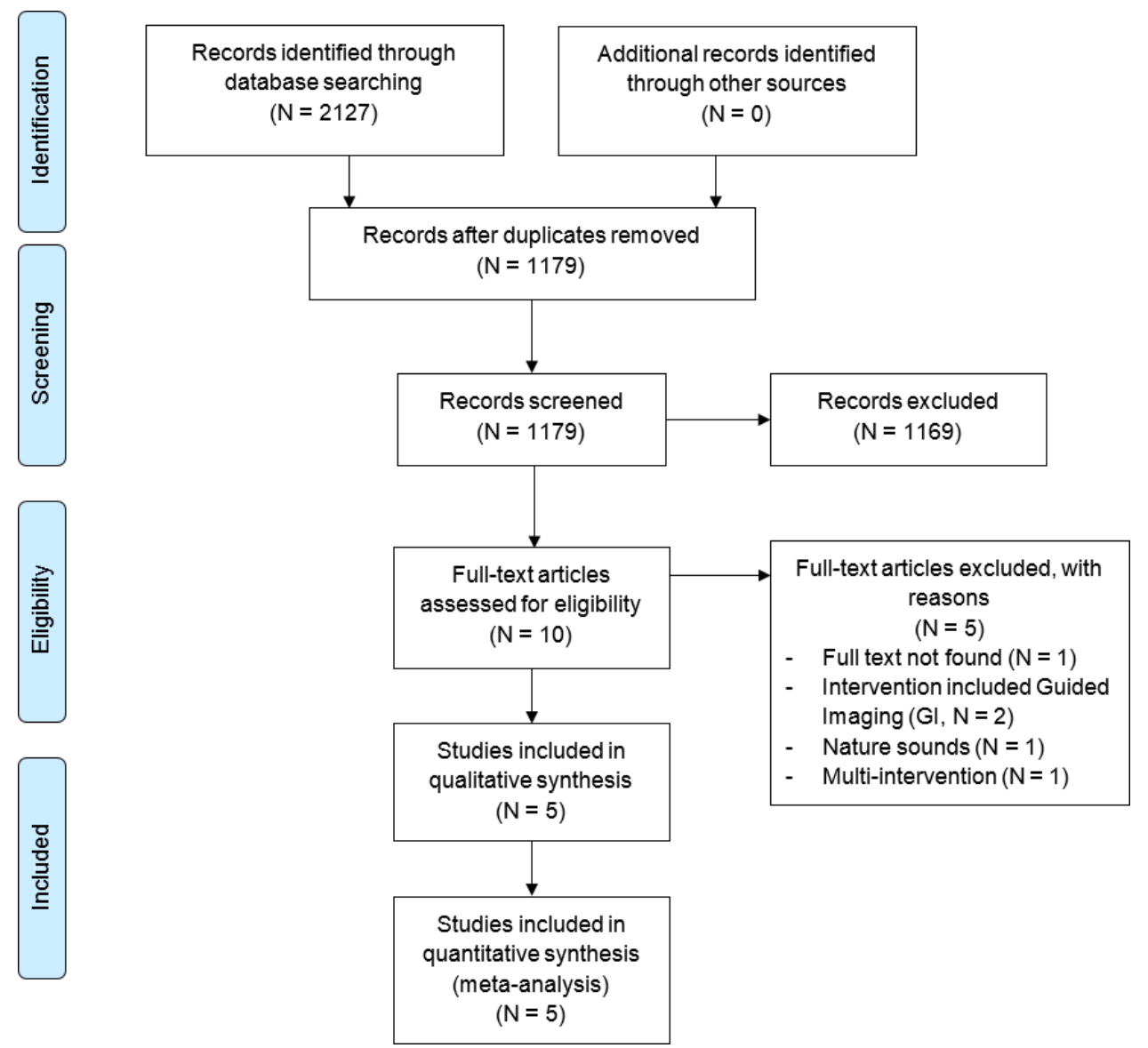

Figure 1 PRISMA flor diagram. N indicates the number of articles. GI, Guided Imaging; PRISMA, Preferred Reporting Items for Systematic Reviews and Meta-Analyses.

evening of intervention. In the study of $\mathrm{Su} e t a l^{51}$ the intervention was only administered in the night of day 3 after admission to the ICU, and sleep was assessed at baseline (PSG and VSH) and PSG during the first 2 hours of sleep on the same night and VSH in the morning after the night of intervention. Hansen et al applied the intervention during the day on day 3 after admission and assessed sleep once immediately afterwards. Zimmerman et $a \tilde{l}^{2}$ applied the intervention on the night of postoperative day (POD) 2 and assessed sleep at baseline and the morning of POD 3. Ryu et a $\tilde{\nu}^{\tilde{0}}$ did not specify on which POD the music intervention and sleep assessment was performed. Baseline sleep assessment was carried out in only three studies; ${ }^{49} 5152$ no differences were found between the study groups in any of the studies based on baseline data. Control groups received either standard care (two studies), scheduled rest (two studies) or standard care with earplugs and eye shields (one study).

\section{Effect of music on subjectively measured sleep quality}

Sleep quality scores of the final music session were pooled. ${ }^{48-52}$ For the data of Cheraghi et $a l_{2}^{49}$ we applied reverse scoring since they used the PSQI, which was the only tool assessing sleep quality as better when scored lower on the scale. Pooling data resulted in an overall significant effect of recorded music on sleep quality in critical care and surgical patients $(\mathrm{SMD}=1.21 \quad(95 \%$ CI
0.50 to 1.91$), \mathrm{p}<0.01)$. Figure $2 \mathrm{~A}, \mathrm{~B}$ presents the forest and funnel plot. The study of Cheraghi et al showed a relative large effect compared with the other studies. Pooled data excluding the study of Cheraghi et al showed that the effect remained significant (SMD $=0.87$ (95\% CI 0.45 to $1.29), \mathrm{p}<0.01$, figure $2 \mathrm{C}$ ).

Since out meta-analysis eventually only included subjectively measures sleep assessment tools, the backtransformation was based on a recent Dutch study that assessed sleep in 194 patients using the RCSQ, which is a validated and reliable sleep quality assessment tool in the critically ill. ${ }^{54}$ The reported median (IQR) for the RCSQ reported in this study was transformed to mean $\pm \mathrm{SD}$ using the methodology of Wan et al. ${ }^{47}$ This resulted in an SD approximation of 2.24. Using the effect size of our metaanalysis, $\mathrm{SMD}=1.21$, this resulted in a reduction of 2.71 (95\% CI 1.12 to 4.28 ) on the RCSQ, which ranges from 0 to 10 . This implies a $27.1 \%$ (95\% CI 11.2 to 42.8 ) increase in sleep quality in the ICU due to a music intervention. In order to check if the effect in our study population approximates the effect calculated previously, we also applied the back-transformation using the pooled RCSQ SDs $(n=51, S D=2.53)$ of the control groups in our study. This resulted in a reduction of 3.06 (95\% CI 1.27 to 4.83 ) on the RCSQ equal to $30.6 \%$ (95\% CI 12.7 to 48.3 ) increase in sleep quality. 


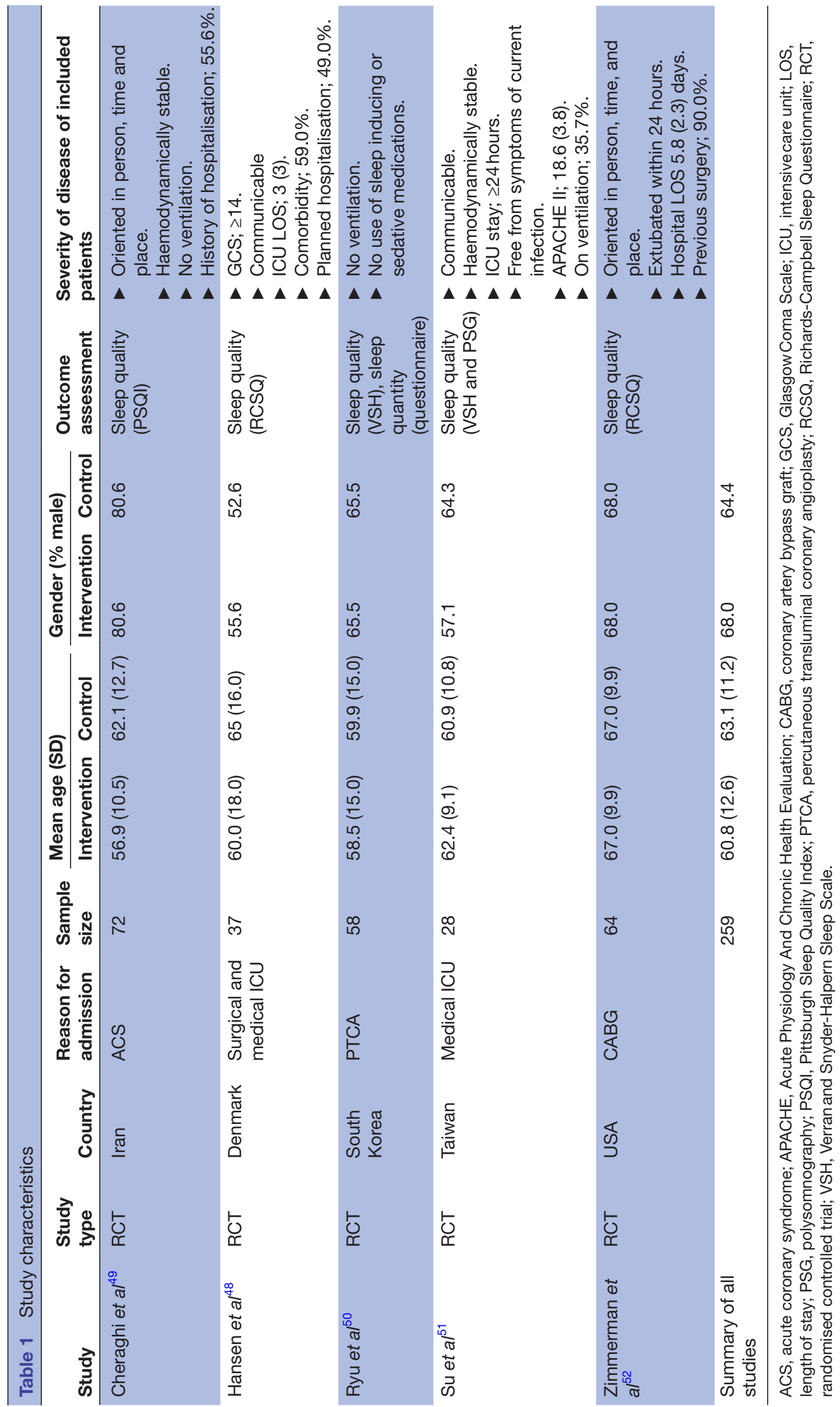




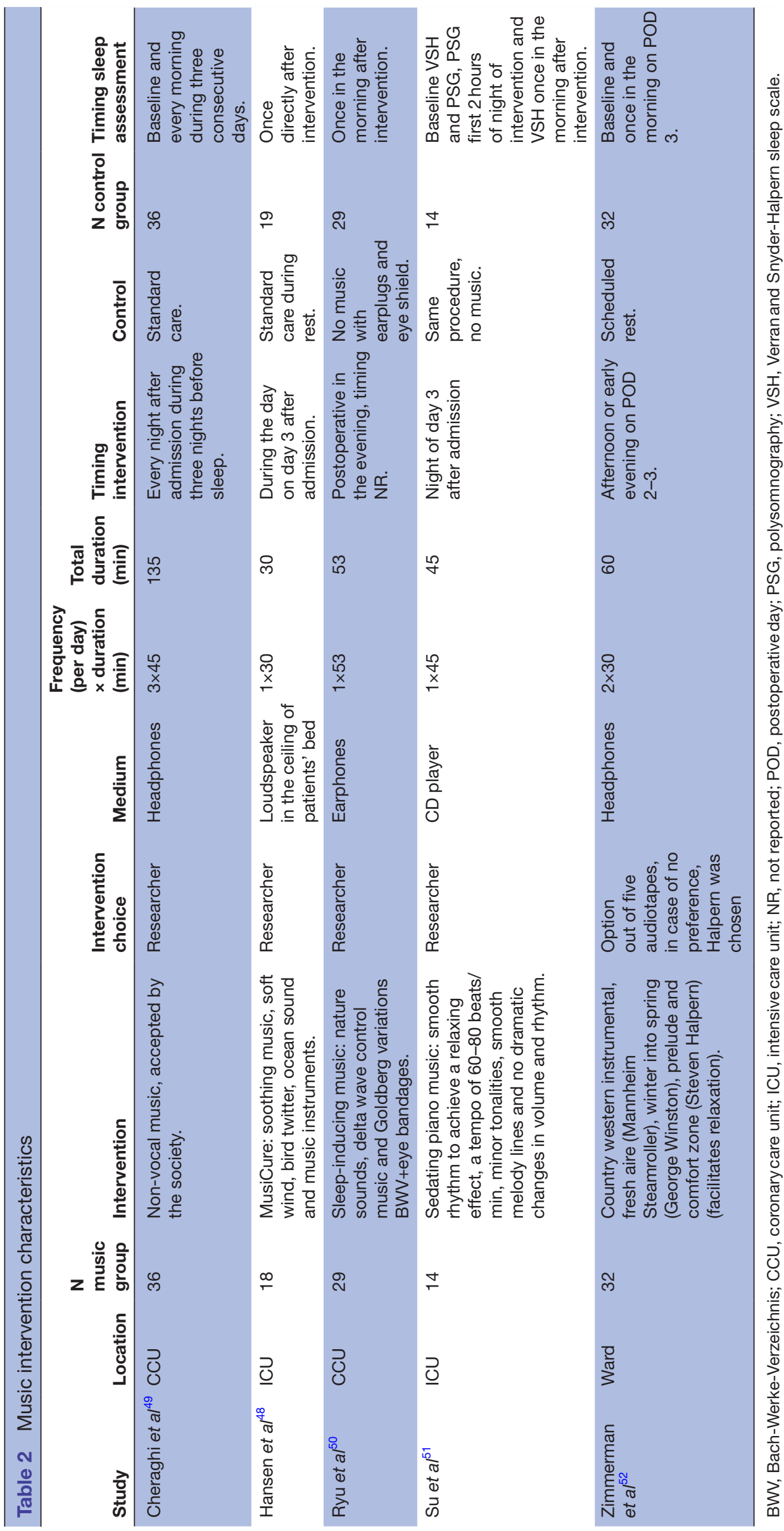




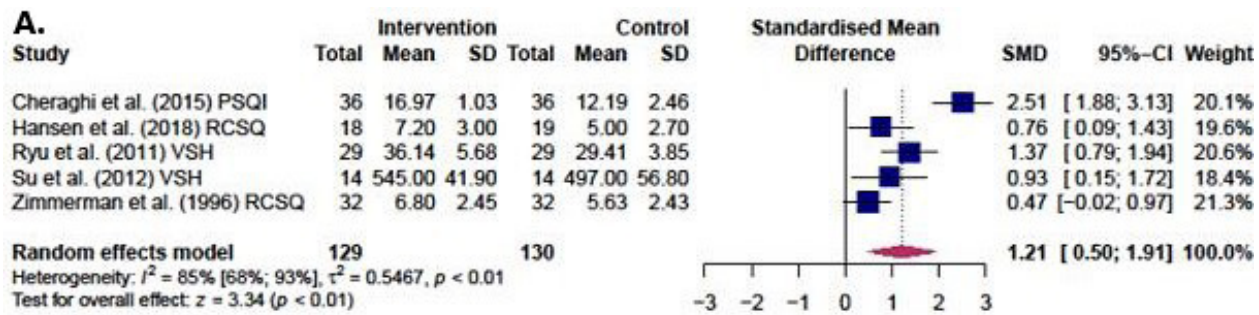

B.
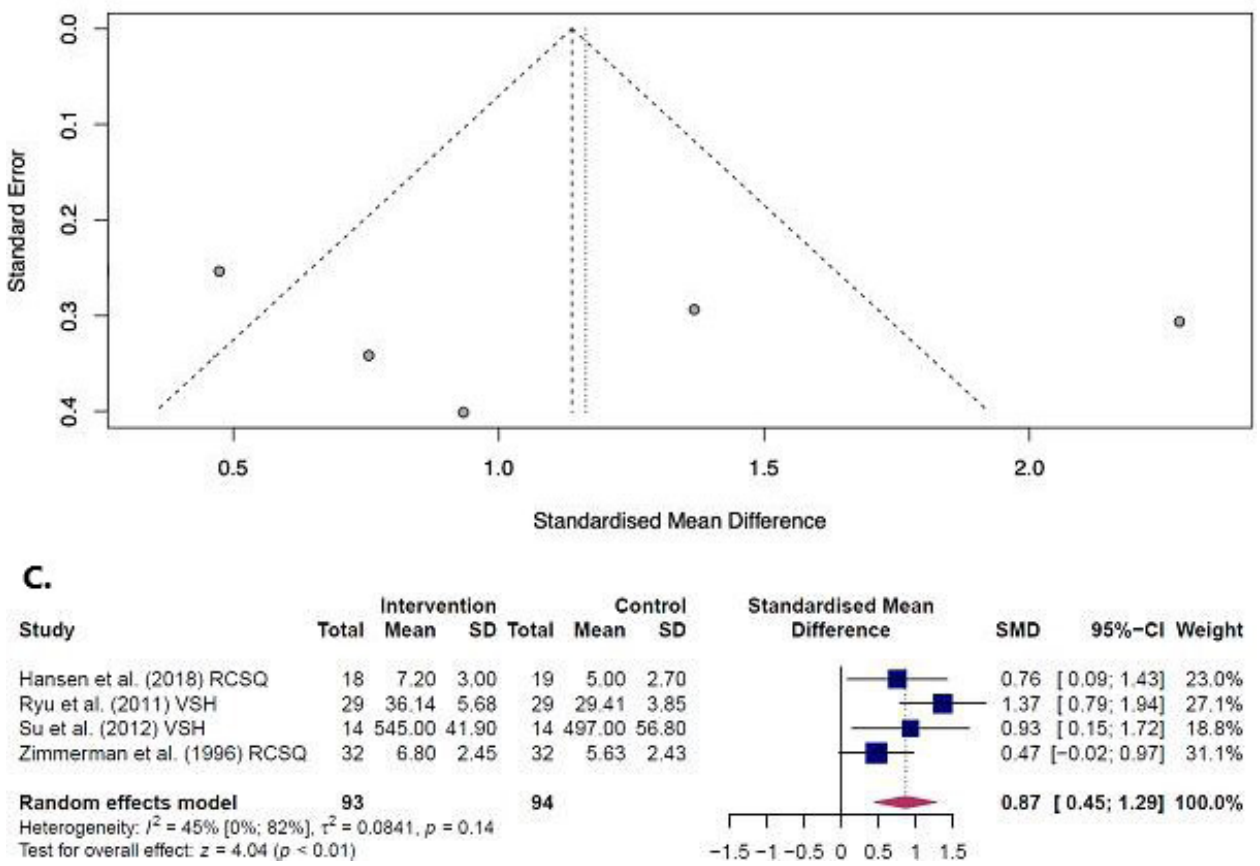

Figure 2 (A) Forest plot assessing the effect of music on subjective sleep quality. (B) Funnel plot assessing the effect of music on subjective sleep quality. (C) Forest plot assessing the effect of music on subjective sleep, excluding the paper of Cheraghi et al. SMD, standardised mean difference.

\section{Effect of music on subjectively measured sleep quantity}

Meta-analysis on the effect of music on sleep quantity could not be performed since it was only assessed by the study of Ryu et $a b^{50}$ in PTCA patients. This study found a statistically significant higher sleep duration in the music group $(n=29)$ compared with the control group $(n=29)$, $279.31 \pm 43.99$ and $243.10 \pm 42.68$, respectively, with a p value of 0.002 .

\section{Effect of music on objectively measured sleep}

As mentioned before, only the study of Su et al measured sleep objectively using the PSG. In this study, they found a significant difference in the N2 (Wald $\chi^{2}=6.03, p=0.014$ ) and N3 (Wald $\chi^{2}=7.02, p=0.008$ ) sleep stages, indicating that the music group had a shorter N2 sleep stage and a longer N3 sleep stage.

\section{Risk of bias assessment}

The overall risk of bias was moderate to high (figure 3). All included studies reported the use of randomisation, but a high risk of selection bias was considered due to insufficient details regarding random sequence generation in two studies ${ }^{48} 52$ and allocation concealment in four studies. ${ }^{4-52}$ Due to the type of intervention and the subjective outcome assessment blinding of participants and personnel was not possible, therefore performance and detection bias were considered high in all studies. Attrition bias was considered low in all studies since none of the studies had a drop-out rate of $\geq 10 \%$. A full study protocol was missing (assessed by checking reported registration number of the trial in the paper and registers for clinical trials (eg, ClinicalTrials.gov) for all included studies leading to an unclear risk for reporting bias. Two included studies (20\%) had a high risk of bias due to other reasons; Cheraghi et $a l^{49}$ excluded patients if listening to the music induced anxiety or brought up bad memories, which could have contributed to an overestimation of the effect of music intervention on sleep quality, and Hansen et $a t^{8}$ measured sleep quality during daytime rest with the $\mathrm{RCSQ}$, which is only a validated measurement tool for sleep quality after nocturnal sleep. Figure 4 presents the summary of the risk of bias assessment.

\section{Grade certainty rating}

Risk of bias was moderate to high for sleep quality. Imprecision was considered medium since the effect size in the 95\% CI of the pooled estimate ranged from medium to 


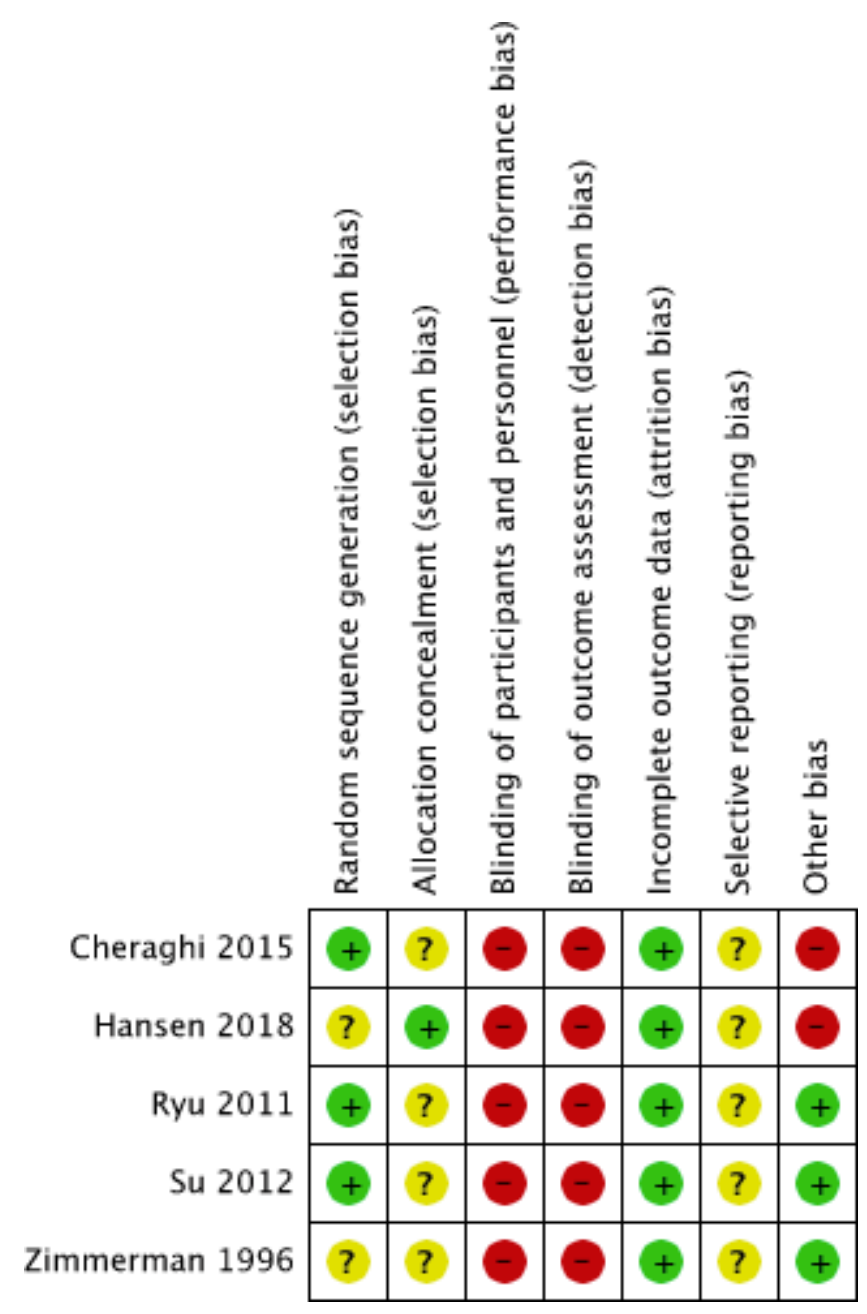

Figure 3 Risk of bias assessment.

high, which could influence clinical decision making. Consistency was considered moderate to high since individual studies included in the meta-analysis showed a positive influence of music on sleep quality, the $95 \%$ CIs overlapped, but the heterogeneity was high. Heterogeneity was moderate to high and statistically significant for sleep quality $\left(\mathrm{I}^{2}=85 \%\right.$ (95\% CI $68 \%$ to $\left.93 \%\right), \mathrm{T}^{2}=0.5$, $\mathrm{p}<0.01$ ), possibly caused by the differences in patient characteristics. After the exclusion of the outlier study of Cheraghi et al from the meta-analysis, heterogeneity decreased and was non-significant $\left(\mathrm{I}^{2}=45 \%(95 \% \mathrm{CI} 0 \%\right.$ to $82 \%)$, $\left.\mathrm{T}^{2}=0.08, \mathrm{p}=0.14\right)$. Meta-regression, to assess the reason for high heterogeneity, could not be performed since there was a limited number of papers available. Directness was considered high as studies directly investigated music in the population of interest, the intervention was mostly applied on the third day after ICU/CCU admission or after surgery and the reported outcomes were critical for decision making (eg, if sleep quality was low, administration of sleep medication could be considered). Scattering in the funnel plot is symmetrical; therefore, the risk for publication bias is considered low. In conclusion, we rate the GRADE certainty rating as moderate.

\section{DISCUSSION}

This meta-analysis showed a significant beneficial effect of recorded music intervention on subjectively measures of sleep quality in critically ill and post-CABG patients admitted to the ward (SMD=1.21 (95\% CI 0.50 to 1.91 ), $\mathrm{p}<0.01$ ), when on average $40 \mathrm{~min}$ (range $30-53$ ) per session/day of music is applied. The overall risk of bias was moderate to high.

These findings are in line with the current literature. ${ }^{29}{ }^{55-57} \mathrm{Hu}$ et $a l^{29}$ reviewed literature for the effect of non-pharmacological interventions on sleep quality in the ICU. Although they could not pool data and their quality of evidence was low, they found that music intervention might positively influence sleep quality. Also, Feng et a $a \tilde{l}^{5}$ studied the effect of music and other non-pharmacological interventions (eg, music combined with other interventions and acupuncture) on primary insomnia (insomnia after ruling out several other conditions such as psychiatric (depression and anxiety), medical (pain), circadian (phase-delay syndrome) or other sleep disorders. They found that solely music intervention had the highest ranking and seemed to offer clear advantages on sleep quality. Our findings are also in line with the current literature on the effect of music on other outcomes in the critically ill population. These studies show effectiveness of music on anxiety, pain, vital parameters, inflammatory markers and medication requirement. ${ }^{40} 4158-60$

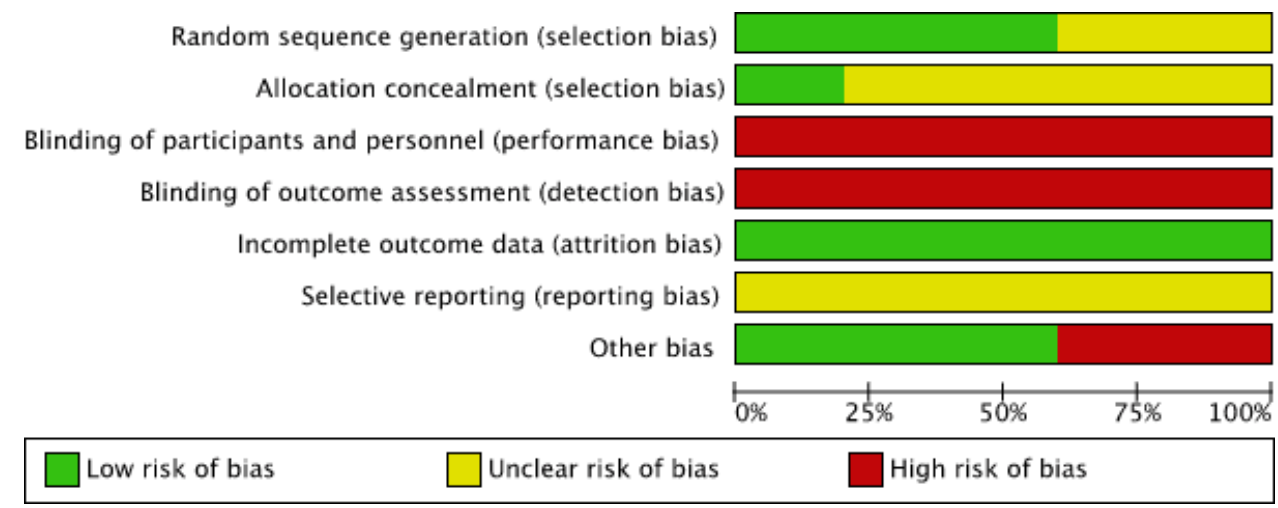

Figure 4 Summary of risk of bias. 
Sleep architecture in critical care patients is characterised by decreased TST, sleep divided into short discrete episodes, sleep occurring mostly during daytime, increased amount of light sleep stages and decreased amount of deeper sleep stage. ${ }^{81761-64}$ This leads to loss of the restorative and resting function of sleep, which is achieved by deeper sleep stages. ${ }^{40} 616365$ Sleep disturbances have been associated with cognitive impairment, including delirium, prolonged duration of mechanical ventilation, altered immune function and long-term psychological comorbidities. ${ }^{81766-69}$ Furthermore, acutely developed sleep disturbances in the ICU can persist after discharge and is the most common stressful factor experienced by patients. ${ }^{61}{ }^{70-73}$ Furthermore, the administration of sedative and analgesic medication, aimed to improve patient comfort, may negatively impact sleep quality. $^{5}$ 10-12 74-78 Therefore, preventing sleep disturbances in critically ill patients is highly important. The one study in our review that assessed sleep objectively found that music significantly increased the deeper sleep stage N3 and decreased the light sleep stage N2, inevitably strengthening the results of our meta-analysis. ${ }^{51}$

We made an approximation of $27.1 \%$ increase in sleep quality if music intervention is applied based on our pooled data. Several studies comparing benzodiazepines to a placebo showed even lower efficacy, ranging from $12.9 \%$ to $21.4 \%$ improvement. ${ }^{79-82}$ This indicates that music may have a similar or even better effect on sleep quality compared with drug therapy with benzodiazepines, while the patient is not exposed to their harmful side effects and risk of dependency. Therefore, the use of recorded music in hospitalised patients can be considered a clinically relevant intervention. Only one paper in this study assessed effect of music on sleep quantity and found a significant increase in sleep duration of $36 \mathrm{~min}$ in a sample of 58 patients. ${ }^{50}$ Krenk et $a l^{81}$ found that zolpidem shortened TST with $28 \mathrm{~min}$ compared with the placebo group; this finding was not significant. Simons et $a l^{82}$ found a significant extension of $57 \mathrm{~min}$ when using temazepam in healthy volunteers. Also, Sharma et al measured an increased TST when zolpidem was used; unfortunately, effects on deeper sleep stages were not found. Of note, extension of the TST does not necessarily lead to better sleep, since better sleep depends more on the proportion of restorative sleep.

The physiology behind the effect of music on sleep remains unclear. Several theories have been proposed on the effect of music on sleep including: rhythmic entrainment, masking and distraction. ${ }^{57}$ It is important to recognise that the effect of music also entails the neurophysiological and psychophysiological levels. ${ }^{83-86}$ Literature indicates that music induces the anxiolytic effect through suppression of the sympathetic nervous system. ${ }^{87} 88$ Also, music stimulates the release of endorphins by activating memory and the limbic system, which plays an important role in emotional well-being. ${ }^{589}$ More specifically, the nucleus accumbens is activated leading to increased dopamine release and deactivation of areas in the brain related to stress and cortisol signalling. ${ }^{90}$ Furthermore, several studies have suggested positive effects of vagus nerve stimulation ${ }^{91}$ on objective and subjective sleep parameters in patients with epilepsy. ${ }^{92}{ }^{93}$ Unfortunately, in spite of these theoretical considerations, we did not find literature on the mechanistic effect of music on sleep architecture. Thus, sleep disturbances are multifactorial, and non-pharmacological interventions should focus on tackling multiple factors (anxiety, pain, stress, etc) affecting sleep at the same time.

The GRADE certainty was rated moderate, which supports the estimated clinical relevant effects. Unfortunately, the relatively small number of studies and sample sizes lead to uncertain reliability and validity of this study, which is also seen in the current literature regarding the effect of music on sleep in other populations. ${ }^{79-81}$

\section{Strengths and limitations}

The sleep assessment tools used in the included studies of this meta-analysis were all validated, reliable and easily applicable. All tools used in the studies included in the meta-analysis are validated and reliable self-reporting questionnaires assessing sleep health of the previous night and are used to assess sleep quality in the studies included in this meta-analysis. The tools are widely used to measure sleep quality in hospitalised patients and thus are deemed conceptually to perform meta-analysis. ${ }^{94}$ An important limitation of this review is the limited amount of studies included for quantitative analysis. Second, heterogeneity was high. Third, there was a moderate to high risk of bias due to insufficient information on random sequence generation and allocation concealment, the primary outcome including subjective patient-reported questionnaires, and due to blinding of participants in a music intervention study not being feasible. Although, Chlan et $a \ell^{55}$ tried to avoid bias due to non-blinding in a large clinical trial on music intervention, that found a decrease in anxiety in the music group, by including an extra control group wearing noise-cancelling headphones (without music).

\section{Future research recommendations}

We suggest future research should consist of high-quality RCTs with the use of objective tools for sleep assessment, as recorded music seems effective and clinically relevant, in order to make more definite conclusions regarding the effect of recorded music on sleep. Since until now relatively small studies with a high variability in the music 'dose' are conducted, we recommend future studies to focus on larger sample sizes with a high methodological quality in order to avoid a substantial risk of bias. Studies with music interventions should report the type of music, timing, duration and frequency of the intervention and sleep assessment in their studies with a validated and reproducible tool. Our analyses suggest that a minimum of $30 \mathrm{~min}$ per day/session is sufficient in order for the music intervention to be effective for the sleep quality. This minimum of $30 \mathrm{~min}$ of music per session/day is 
also consistent with the current literature. ${ }^{37} 4059$ Previous studies can serve as a guide for future studies. ${ }^{96}$

\section{Conclusion}

This systematic review and meta-analysis of randomised controlled trials showed that recorded music interventions significantly increase sleep quality in the critically ill, ACS or PTCA patients and after cardiac surgery for coronary artery disease. Music is easily applicable and has no risks and side effects and should therefore be considered as a suitable non-medicinal alternative for sleep quality improvement in these patient groups. Since the clinical trials performed until now are small and of low quality, we suggest larger and high-quality randomised clinical trials for future research, including broader patient populations.

Acknowledgements The authors would like to thank W Bramer, biomedical information specialist of the Medical Library at the Erasmus University Medical Centre, for his assistance in the literature search and $\mathrm{J}$ van Rosmalen, assistant professor at the Department of Biostatistics of the Erasmus University Medical Centre, for his assistance in the statistical analysis.

Contributors $\mathrm{JJ}$ conceived the study idea. EK and EV coordinated the systematic review. EK and EV screened abstracts and full texts. EK and EV wrote the first draft of the manuscript and judged risk of bias in the studies. EK, EV, MvdJ, MK and JJ interpreted the data. EK, EV, MvdJ, MK and JJ critically revised the manuscript. EK, $\mathrm{EV}, \mathrm{MvdJ}, \mathrm{MK}$ and JJ had full access to all of the data in the study and can take responsibility for the integrity of the data and the accuracy of the data analysis.

Funding The authors have not declared a specific grant for this research from any funding agency in the public, commercial or not-for-profit sectors.

Competing interests None declared.

Patient consent for publication Not required

Provenance and peer review Not commissioned; externally peer reviewed.

Data availability statement Data sharing not applicable as no datasets generated and/or analysed for this study.

Supplemental material This content has been supplied by the author(s). It has not been vetted by BMJ Publishing Group Limited (BMJ) and may not have been peer-reviewed. Any opinions or recommendations discussed are solely those of the author(s) and are not endorsed by BMJ. BMJ disclaims all liability and responsibility arising from any reliance placed on the content. Where the content includes any translated material, BMJ does not warrant the accuracy and reliability of the translations (including but not limited to local regulations, clinical guidelines, terminology, drug names and drug dosages), and is not responsible for any error and/or omissions arising from translation and adaptation or otherwise.

Open access This is an open access article distributed in accordance with the Creative Commons Attribution 4.0 Unported (CC BY 4.0) license, which permits others to copy, redistribute, remix, transform and build upon this work for any purpose, provided the original work is properly cited, a link to the licence is given, and indication of whether changes were made. See: https://creativecommons.org/ licenses/by/4.0/.

ORCID iDs

Ellaha Kakar http://orcid.org/0000-0002-7472-308X

Markus Klimek http://orcid.org/0000-0002-0122-9929

\section{REFERENCES}

1 Dobing S, Frolova N, McAlister F, et al. Sleep quality and factors influencing self-reported sleep duration and quality in the general internal medicine inpatient population. PLoS One 2016;11:e0156735.

2 Doğan O, Ertekin Şükran, Doğan S. Sleep quality in hospitalized patients. J Clin Nurs 2005;14:107-13.

3 Jakobsen G, Engstrøm M, Thronæs M, et al. Sleep quality in hospitalized patients with advanced cancer: an observational study using self-reports of sleep and actigraphy. Support Care Cancer 2020:28:2015-23.

4 Park MJ, Kim KH. What affects the subjective sleep quality of hospitalized elderly patients? Geriatr Gerontol Int 2017;17:471-9.

5 Wesselius HM, van den Ende ES, Alsma J, et al. Quality and quantity of sleep and factors associated with sleep disturbance in hospitalized patients. JAMA Intern Med 2018;178:1201-8.

6 Cilingir D, Hintistan S, Ergene O. Factors affecting the sleep status of surgical and medical patients at a university hospital of turkey. J Pak Med Assoc 2016;66:1535-40.

7 Bani Younis M, Hayajneh FA. Quality of sleep among intensive care unit patients: a literature review. Crit Care Nurs Q 2018;41:170-7.

8 Pulak LM, Jensen L. Sleep in the intensive care unit: a review. J Intensive Care Med 2016;31:14-23.

9 Parthasarathy S, Friese RS, Ayas NT. Biological validity to sleep measurements during critical illness*. Crit Care Med 2010;38:705-6.

10 DeMartinis NA, Kamath J, Winokur A. New approaches for the treatment of sleep disorders. Adv Pharmacol 2009;57:187-235.

11 Yilmaz M, Sayin Y, Gurler H. Sleep quality of hospitalized patients in surgical units. Nurs Forum 2012;47:183-92.

12 Klemann N, Hansen MV, Gögenur I. Factors affecting post-operative sleep in patients undergoing colorectal surgery - a systematic review. Dan Med J 2015;62:A5053.

13 Su X, Wang DX. Improve postoperative sleep: what can we do? Curr Opin Anaesthesiol 2018;31:83-8.

14 Kjølhede P, Langström P, Nilsson P, et al. The impact of quality of sleep on recovery from fast-track abdominal hysterectomy. J Clin Sleep Med 2012;08:395-402.

15 Rosenberg J. Sleep disturbances after non-cardiac surgery. Sleep Med Rev 2001;5:129-37.

16 Gögenur I. Postoperative circadian disturbances. Dan Med Bull 2010;57:B4205.

17 Devlin JW, Skrobik Y, Gélinas C, et al. Clinical practice guidelines for the prevention and management of pain, Agitation/Sedation, delirium, immobility, and sleep disruption in adult patients in the ICU. Crit Care Med 2018;46:e825-73.

18 Lieberman HR, Bathalon GP, Falco CM, et al. Severe decrements in cognition function and mood induced by sleep loss, heat, dehydration, and undernutrition during simulated combat. Biol Psychiatry 2005;57:422-9.

19 Telias I, Wilcox ME. Sleep and circadian rhythm in critical illness. Crit Care 2019;23:82.

20 Peever J, Fuller PM. The biology of REM sleep. Curr Biol 2017;27:R1237-48.

21 Gillis CM, Poyant JO, Degrado JR, et al. Inpatient pharmacological sleep aid utilization is common at a tertiary medical center. $J$ Hosp Med 2014;9:652-7.

22 Frighetto L, Marra C, Bandali S, et al. An assessment of quality of sleep and the use of drugs with sedating properties in hospitalized adult patients. Health Qual Life Outcomes 2004;2:17.

23 van Beek S, Kroon J, Rijs K. The effect of midazolam as premedication on the quality of postoperative recovery after laparotomy: a randomized clinical trial Effet du midazolam en premedication sur la qualite de la recuperation postoperatoire apres une laparotomie : une etude clinique randomisee. Can J Anaesth 2020;67:32-41.

24 Maurice-Szamburski A, Auquier P, Viarre-Oreal V, et al. Effect of sedative premedication on patient experience after general anesthesia: a randomized clinical trial. JAMA 2015;313:916-25.

25 Mijderwijk H, van Beek S, Klimek M, et al. Lorazepam does not improve the quality of recovery in day-case surgery patients: a randomised placebo-controlled clinical trial. Eur J Anaesthesiol 2013;30:743-51.

26 Halfens R, Cox K, Kuppen-Van Merwijk A. Effect of the use of sleep medication in Dutch hospitals on the use of sleep medication at home. J Adv Nurs 1994;19:66-70.

27 Brummett CM, Waljee JF, Goesling J, et al. New persistent opioid use after minor and major surgical procedures in US adults. JAMA Surg 2017:152:e170504.

28 Lenhart SE, Buysse DJ. Treatment of insomnia in hospitalized patients. Ann Pharmacother 2001;35:1449-57.

29 Hu R-F, Jiang X-Y, Chen J, et al. Non-Pharmacological interventions for sleep promotion in the intensive care unit. Cochrane Database Syst Rev 2015;10:CD008808.

30 Brandt J, Leong C. Benzodiazepines and Z-Drugs: an updated review of major adverse outcomes reported on in epidemiologic research. Drugs R D 2017;17:493-507.

31 Glass J, Lanctôt KL, Herrmann N, et al. Sedative hypnotics in older people with insomnia: meta-analysis of risks and benefits. BMJ 2005;331:1169. 
32 Weinbroum AA, Szold O, Ogorek D, et al. The midazolam-induced paradox phenomenon is reversible by flumazenil. epidemiology, patient characteristics and review of the literature. Eur $J$ Anaesthesiol 2001:18:789-97.

33 The American Geriatrics Society Expert Panel on Postoperative Delirium in Older Adults. American geriatrics Society abstracted clinical practice guideline for postoperative delirium in older adults. J Am Geriatr Soc 2015;63:142-50.

34 Bringman $\mathrm{H}$, Giesecke $\mathrm{K}$, Thörne $\mathrm{A}$, et al. Relaxing music as premedication before surgery: a randomised controlled trial. Acta Anaesthesiol Scand 2009;53:759-64.

35 Kassie GM, Nguyen TA, Kalisch Ellett LM, et al. Preoperative medication use and postoperative delirium: a systematic review. BMC Geriatr 2017;17:298.

36 Zaal IJ, Devlin JW, Hazelbag M, et al. Benzodiazepine-associated delirium in critically ill adults. Intensive Care Med 2015;41:2130-7.

37 Fu VX, Oomens P, Klimek M, et al. The effect of perioperative music on medication requirement and hospital length of stay: a metaanalysis. Ann Surg 2020;272:961-972.

38 Fu VX, Oomens P, Sneiders D, et al. The effect of perioperative music on the stress response to surgery: a meta-analysis. J Surg Res 2019;244:444-55.

39 Kühlmann AYR, de Rooij A, Kroese LF, et al. Meta-analysis evaluating music interventions for anxiety and pain in surgery. Br J Surg 2018;105:773-83.

40 Bradt J, Dileo C. Music interventions for mechanically ventilated patients. Cochrane Database Syst Rev 2014;12:CD006902.

41 Khan SH, Kitsis M, Golovyan D, et al. Effects of music intervention on inflammatory markers in critically ill and post-operative patients: a systematic review of the literature. Heart Lung 2018;47:489-96.

42 Moher D, Liberati A, Tetzlaff J, et al. Preferred reporting items for systematic reviews and meta-analyses: the PRISMA statement. PLOS Med 2009;6:e1000097.

43 Bramer WM, Rethlefsen ML, Mast F, et al. Evaluation of a new method for librarian-mediated literature searches for systematic reviews. Res Synth Methods 2018;9:510-20.

44 Schardt C, Adams MB, Owens T, et al. Utilization of the PICO framework to improve searching PubMed for clinical questions. BMC Med Inform Decis Mak 2007;7:16.

45 Higgins JPT GS. The Cochrane handbook for systematic reviews of interventions version 5.1.0, 2011.

46 Guyatt GH, Oxman AD, Vist GE, et al. Grade: an emerging consensus on rating quality of evidence and strength of recommendations. $B M J$ 2008;336:924-6.

47 Wan X, Wang W, Liu J, et al. Estimating the sample mean and standard deviation from the sample size, median, range and/or interquartile range. BMC Med Res Methodol 2014;14:135.

48 Hansen IP, Langhorn L, Dreyer P. Effects of music during daytime rest in the intensive care unit. Nurs Crit Care 2018;23:207-13.

49 Cheraghi M, Haghani $\mathrm{H}$, Akbari K. The effect of instrumental music on sleep in patients admitted to coronary care unit. Iranian JCard Nurs 2015

50 Ryu M-J, Park JS, Park H. Effect of sleep-inducing music on sleep in persons with percutaneous transluminal coronary angiography in the cardiac care unit. J Clin Nurs 2012;21:728-35.

51 Su C-P, Lai H-L, Chang E-T, et al. A randomized controlled trial of the effects of listening to non-commercial music on quality of nocturnal sleep and relaxation indices in patients in medical intensive care unit. $J$ Adv Nurs 2013;69:1377-89.

52 Zimmerman L, Nieveen J, Barnason S, et al. The effects of music interventions on postoperative pain and sleep in coronary artery bypass graft (CABG) patients. Sch Inq Nurs Pract 1996;10:153-70.

53 Morgenthaler TI, Lee-Chiong T, Alessi C, et al. Practice parameters for the clinical evaluation and treatment of circadian rhythm sleep disorders. An American Academy of sleep medicine report. Sleep 2007;30:1445-59.

54 Rood P, Frenzel T, Verhage R, et al. Development and daily use of a numeric rating score to assess sleep quality in ICU patients. $J$ Crit Care 2019;52:68-74.

55 Feng F, Zhang Y, Hou J, et al. Can music improve sleep quality in adults with primary insomnia? A systematic review and network meta-analysis. Int J Nurs Stud 2018;77:189-96.

56 Bani Younis M, Hayajneh F, Batiha A-M. Measurement and nonpharmacologic management of sleep disturbance in the intensive care units. Crit Care Nurs Q 2019;42:75-80.

57 Dickson GT, Schubert E. How does music aid sleep? literature review. Sleep Med 2019;63:142-50.

58 Beaulieu-Boire G, Bourque S, Chagnon F, et al. Music and biological stress dampening in mechanically-ventilated patients at the intensive care unit ward-a prospective interventional randomized crossover trial. J Crit Care 2013;28:442-50.
59 Richard-Lalonde M, Gélinas C, Boitor M, et al. The effect of music on pain in the adult intensive care unit: a systematic review of randomized controlled trials. J Pain Symptom Manage 2020;59:1304-19.

60 Umbrello M, Sorrenti T, Mistraletti G, et al. Music therapy reduces stress and anxiety in critically ill patients: a systematic review of randomized clinical trials. Minerva Anestesiol 2019;85:886-98.

61 Pisani MA, Friese RS, Gehlbach BK, et al. Sleep in the intensive care unit. Am J Respir Crit Care Med 2015;191:731-8.

62 Cooper AB, Thornley KS, Young GB, et al. Sleep in critically ill patients requiring mechanical ventilation. Chest 2000;117:809-18.

63 Freedman NS, Gazendm J, Levan L, et al. Abnormal sleep/ wake cycles and the effect of environmental noise on sleep disruption in the intensive care unit. Am J Respir Crit Care Med 2001;163:451-7.

64 Elliott R, McKinley S, Cistulli P, et al. Characterisation of sleep in intensive care using 24-hour polysomnography: an observational study. Crit Care 2013;17:R46.

65 Boyko Y, Jennum P, Toft P. Sleep quality and circadian rhythm disruption in the intensive care unit: a review. Nat Sci Sleep 2017;9:277-84.

66 Sanders RD, Maze M. Contribution of sedative-hypnotic agents to delirium via modulation of the sleep pathway. Can $J$ Anaesth 2011;58:149-56.

67 Friese RS. Sleep and recovery from critical illness and injury: a review of theory, current practice, and future directions. Crit Care Med 2008;36:697-705.

68 Opp MR. Sleep and psychoneuroimmunology. Immunol Allergy Clin North Am 2009;29:295-307.

69 Orwelius L, Nordlund A, Nordlund P, et al. Prevalence of sleep disturbances and long-term reduced health-related quality of life after critical care: a prospective multicenter cohort study. Crit Care 2008;12:R97.

70 Tembo AC, Parker V, Higgins I. The experience of sleep deprivation in intensive care patients: findings from a larger hermeneutic phenomenological study. Intensive Crit Care Nurs 2013;29:310-6.

71 Simini B. Patients' perceptions of intensive care. Lancet 1999;354:571-2.

72 Rotondi AJ, Chelluri L, Sirio C, et al. Patients' recollections of stressful experiences while receiving prolonged mechanical ventilation in an intensive care unit. Crit Care Med 2002;30:746-52.

73 Kamdar BB, Needham DM, Collop NA. Sleep deprivation in critical illness: its role in physical and psychological recovery. $J$ Intensive Care Med 2012;27:97-111.

74 Barr J, Fraser GL, Puntillo K, et al. Clinical practice guidelines for the management of pain, agitation, and delirium in adult patients in the intensive care unit. Crit Care Med 2013;41:263-306.

75 Hetland B, Lindquist R, Chlan LL. The influence of music during mechanical ventilation and weaning from mechanical ventilation: a review. Heart \& Lung 2015;44:416-25.

76 Jean R, Shah P, Yudelevich E, et al. Effects of deep sedation on sleep in critically ill medical patients on mechanical ventilation. J Sleep Res 2020;29:e12894.

77 Pandharipande P, Ely EW. Sedative and analgesic medications: risk factors for delirium and sleep disturbances in the critically ill. Crit Care Clin 2006;22:313-27.

78 Dolan R, Huh J, Tiwari N, et al. A prospective analysis of sleep deprivation and disturbance in surgical patients. Ann Med Surg 2016;6:1-5.

79 Sharma MK, Kainth S, Kumar S, et al. Effects of zolpidem on sleep parameters in patients with cirrhosis and sleep disturbances: a randomized, placebo-controlled trial. Clin Mol Hepatol 2019;25:199-209.

80 Huang Y, Mai W, Cai X, et al. The effect of zolpidem on sleep quality, stress status, and nondipping hypertension. Sleep Med 2012;13:263-8.

81 Krenk L, Jennum P, Kehlet H. Postoperative sleep disturbances after zolpidem treatment in fast-track hip and knee replacement. J Clin Sleep Med 2014;10:321-6.

82 Simons R, Koerhuis CL, Valk PJL, et al. Usefulness of temazepam and zaleplon to induce afternoon sleep. Mil Med 2006;171:998-1001.

83 Koelsch S, Fritz T, Schulze K, et al. Adults and children processing music: an fMRI study. Neuroimage 2005;25:1068-76.

84 Menon V, Levitin DJ. The rewards of music listening: response and physiological connectivity of the mesolimbic system. Neuroimage 2005;28:175-84.

85 Koelsch S. A coordinate-based meta-analysis of music-evoked emotions. Neuroimage 2020;223:117350.

86 Koelsch S. Brain correlates of music-evoked emotions. Nat Rev Neurosci 2014;15:170-80. 
87 Chlan L. Effectiveness of a music therapy intervention on relaxation and anxiety for patients receiving ventilatory assistance. Heart \& Lung 1998;27:169-76.

88 Gillen E, Biley F, Allen D. Effects of music listening on adult patients' pre-procedural state anxiety in hospital. JBI Libr Syst Rev 2008;6:686-732.

89 Tracy MF, Chlan L. Nonpharmacological interventions to manage common symptoms in patients receiving mechanical ventilation. Crit Care Nurse 2011;31:19-28.

90 Chanda ML, Levitin DJ. The neurochemistry of music. Trends Cogn Sci 2013;17:179-93.

91 Sigurdardóttir GA, Nielsen PM, Rønager J, et al. A pilot study on high amplitude low frequency-music impulse stimulation as an add-on treatment for depression. Brain Behav 2019;9:e01399.

92 Jain SV, Glauser TA. Effects of epilepsy treatments on sleep architecture and daytime sleepiness: an evidence-based review of objective sleep metrics. Epilepsia 2014;55:26-37.
93 Romero-Osorio Óscar, Gil-Tamayo S, Nariño D, et al. Changes in sleep patterns after vagus nerve stimulation, deep brain stimulation or epilepsy surgery: systematic review of the literature. Seizure 2018;56:4-8.

94 Hoey LM, Fulbrook P, Douglas JA. Sleep assessment of hospitalised patients: a literature review. Int J Nurs Stud 2014;51:1281-8.

95 Chlan LL, Weinert CR, Heiderscheit A, et al. Effects of patientdirected music intervention on anxiety and sedative exposure in critically ill patients receiving mechanical ventilatory support: a randomized clinical trial. JAMA 2013;309:2335-44.

96 Billar RJ, KühImann AYR, Schnater JM, et al. Interventions with music in pectus excavatum treatment (IMPECT trial): a study protocol for a randomised controlled trial investigating the clinical effects of perioperative music interventions. BMJ Open 2020;10:e036380. 